\title{
Bone cement implantation syndrome: An unavoidable catastrophe?
}

\author{
Vera Barbosa, Sandra Carneiro, Joana Barros, Teresa Rebelo
}

\begin{abstract}
Introduction: Bone cement implantation syndrome is an important cause of perioperative morbidity and mortality (0.6-1\%) in patients undergoing cemented hip arthroplasty. Clinical features include cardiac, neurologic and respiratory impairments that may lead to cardiac arrest. It has been proposed that the effect of systemically absorbed methyl methacrylate and release of endogenous mediators can be the main process which leads to multiorgan dysfunction. Case Report: We describe a case of bone cement implantation syndrome developed in a hip arthroplasty with a catastrophic outcome, resulting in the death of the patient in 24h. Conclusion: The review of clinical cases of bone cement implantation syndrome is essential in clinical practice given that early recognition and management of this syndrome may improve the overall prognosis.
\end{abstract}

Keywords: Arthroplasty, Cardiac arrest, Cement, Embolism

Vera Barbosa ${ }^{1}$, Sandra Carneiro ${ }^{1}$, Joana Barros ${ }^{2}$, Teresa Rebelo ${ }^{1}$

Affiliations: ${ }^{1}$ Departmento de Anestesiologia, Unidade Local de Saúde de Matosinhos E.P.E., Hospital Pedro Hispano; ${ }^{2}$ Departmento da Mulher, da Criança e do Jovem, Unidade Local de Saúde de Matosinhos E.P.E., Hospital Pedro Hispano.

Corresponding Author: Vera Barbosa, Departmento de Anestesiologia, Unidade Local de Saúde de Matosinhos E.P.E., Hospital Pedro Hispano.

Received: 19 July 2016

Accepted: 03 September 2016

Published: 28 September 2016

\section{How to cite this article}

Barbosa V, Carneiro S, Barros J, Rebelo T. Bone cement implantation syndrome: An unavoidable catastrophe? Case Rep Int 2016;5:40-43.

Article ID: 100029 CRINTVP2016

$$
* * * * * * * * *
$$

doi:10.5348/crint-2016-29-CR-10

\section{INTRODUCTION}

Bone cement implantation syndrome (BCIS) is a poorly defined entity, responsible for significant perioperative morbidity and mortality (0.6-1\%) [1, 2] in patients undergoing cemented hip arthroplasty. According to literature, it may include respiratory (hypoxia, pulmonary hypertension, pulmonary edema and bronchospasm), hematologic (thrombocytopenia), cardiovascular (hypotension, arrhythmia and cardiac arrest) and neurologic dysfunction (loss of consciousness) [3]. The etiology and pathophysiology of BCIS are not fully established [1], although several mechanisms have been proposed such as toxic effect of systemically absorbed methyl methacrylate, fat and bone marrow embolism, use of high pressure during cementing, exothermic reaction, anaphylactic reaction, and release of endogenous mediators (histamine, complement activation, vasodilator cannabinoids) $[4,5]$. In this article, we report a case of BCIS relating to a cemented hip arthroplasty, with catastrophic outcome, resulting in the death of the patient. 


\section{CASE REPORT}

A 73-year-old woman was admitted into the emergency department after a mechanical fall, with a diagnosis of left femur neck fracture, and was proposed for total hip replacement. The fracture (Figure 1) occurred approximately eight hours before the surgery.

Aside from essential hypertension, the patient had no other comorbidities and was independent in her daily activities. The patient had good functional capacity with more than four metabolic equivalents in exercise testing, and there were no previous signs of important cardiorespiratory compromise.

Preoperative investigation, namely, renal function, ionogram, coagulation and hemogram were within normal limits as well as the electrocardiogram (ECG). On the day of the surgery, the patient was hemodynamically stable having peripheral oxygen saturation $\left(\mathrm{SpO}_{2}\right)$ of $99 \%$ on room air. The patient was administered $1 \mathrm{mg}$ of diazepam on the night before the surgery and also on the day of the surgery. Antihypertensive medication was stopped on the day of the surgery.

The patient was placed in right side decubitus after standard monitoring with oximetry, ECG and noninvasive arterial blood pressure, and Ringer's solution was administered.

Subarachnoid block was performed using a 25-gauge Whitacre needle in the L4-L5 interspace. Levobupivacaine $0.5 \%(8 \mathrm{mg})$ and $0.002 \mathrm{mg}$ of sufentanil were injected after free flow of cerebrospinal fluid. Sensory analgesia up to T8-T1o dermatome was confirmed. Hemodynamic parameters remained stable following subarachnoid block.

Intravenous cefazolin (2 g) was administered for surgical infection prophylaxis and the surgery was started.

During the surgery the patient's hemodynamic parameters were stable with pulse rate varying between $64-82 /$ min with sinus rhythm, systolic blood pressure between 108-122 mmHg and diastolic blood pressure between 68-85 mmHg, $\mathrm{SpO}_{2} \geq 98 \%$ (room air) and normal state of consciousness, until just before the bone cement placement.

Seconds after the placement of methyl methacrylate (bone cement) there was a sudden decrease of level of consciousness, from Glasgow Coma Scale 15-7, accompanied by hypotension $(83 / 45 \mathrm{mmHg})$, sinus tachycardia (130 bpm) and desaturation (from 99\% at $21 \%$ of oxygen to $82 \%$ with high flow of oxygen). Pulmonary auscultation was normal, intraoperatory ECG showed sinus tachycardia and arterial blood gas analysis showed metabolic acidosis and hypoxemia. The surgery was stopped immediately.

Orotracheal intubation and mechanical ventilation with $100 \%$ of oxygen was performed. Aggressive fluid therapy and bolus of ephedrine (total of $20 \mathrm{mg}$ ) were administered without consistent improvement and hence an intravenous infusion of norepinephrine (0.15 $\mathrm{\mu g} /$ $\operatorname{kgmin}^{-1}$ ) was started with hemodynamic improvement that allowed the surgery to proceed.

The surgery lasted for 80 minutes (Figure 2). The patient's estimated blood loss was $250 \mathrm{~mL}$, receiving a total of $2.5 \mathrm{~L}$ of crystalloids without the need of blood transfusion. At the end of the surgery the patient was transferred to the intensive care unit (ICU).

In the ICU, laboratory investigation, brain computed tomography, cervical Doppler, transcranial ultrasound and computed tomography pulmonary angiogram revealed no acute abnormalities.

Despite the support provided in the ICU, a progressive deterioration of the general status resulted in the patient's death $24 \mathrm{~h}$ after the surgery.

\section{DISCUSSION}

Hemodynamic effects of pulmonary emboli and sudden increase in pulmonary vascular resistance (PVR) associated with the use of cement has been described

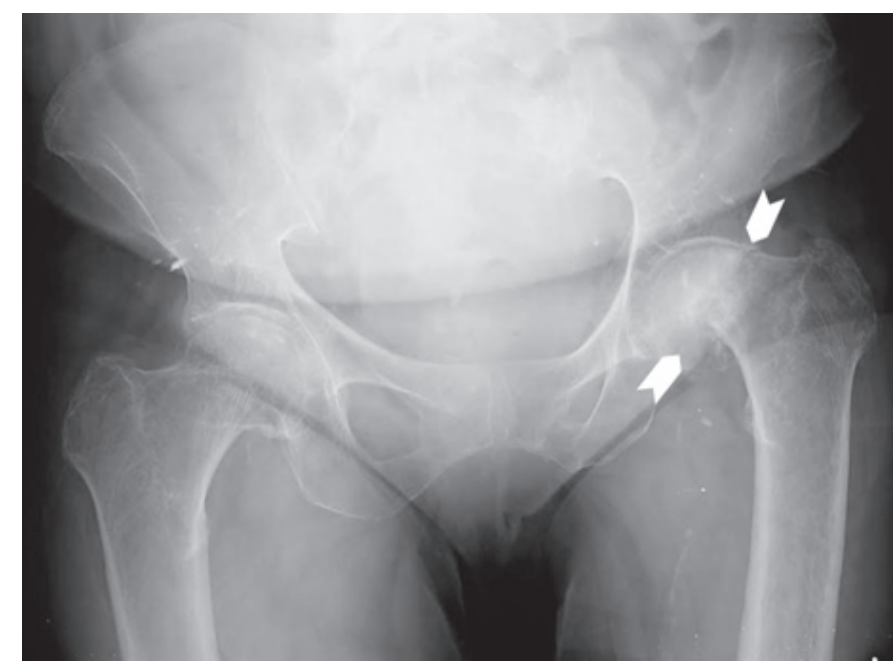

Figure 1: Anteroposterior X-ray film displaying a left femoral neck fracture (arrows).

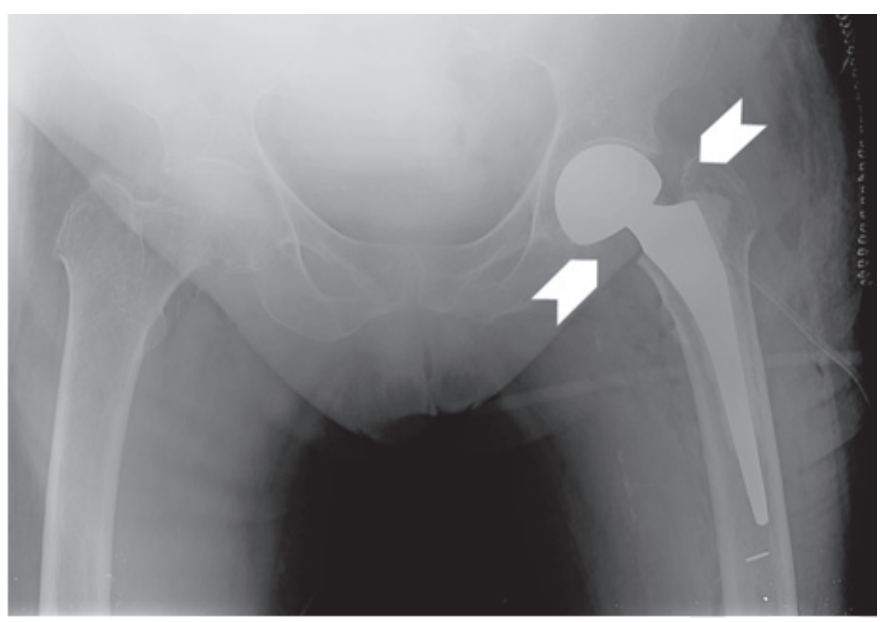

Figure 2: Anteroposterior left hip X-ray displaying left total hip replacement in situ (arrows). 
to be the key feature of BCIS [5]. Embolization usually results from pressurization of bone cavity (often $>300$ $\mathrm{mmHg}$ ) during cementing and prosthesis insertion, with release of vasoactive mediators in pulmonary circulation and increase of the PVR $[1,2,5]$.

Clinical deterioration which can occur within seconds to minutes of cement implantation $[1,5]$ is the result of right ventricular (RV) failure due to high PVR which leads to a reduced cardiac output. Acutely increased $\mathrm{RV}$ pressure promotes dilatation of $\mathrm{RV}$ which pushes the intraventricular septum to the left, leading to poor filling of left ventricle, reduction in cardiac output, systemic hypotension, decrease in coronary perfusion pressure resulting in ischemia and may result in cardiac arrest $[1,2,5]$. It has also been proposed that emboli can reach cerebral circulation through patent foramen ovale or pulmonary, circulation which can be seen by transesophageal echocardiography and transcranial Doppler [5]. Embolization cannot explain all features of BCIS and other mechanisms can be involved, such as histamine release, complement activation and endogenous cannabinoid-mediated vasodilatation [2].

There are some high-risk patients and surgical criteria for developing of BCIS. In 2015, a guideline was published in order to reduce the risk from cemented arthroplasty for hip fracture [6]. Increased patient's age, higher grade of American Society of Anesthesiologists physical grade (III or IV), presence of significant cardiopulmonary disease, use of diuretics, male sex, presence of osteoporosis, malignant disease, revision surgery, surgery in uninstrumented femur, use of excessive pressure during cementing and use of longstem prosthesis are some of the factors that may increase the risk of developing BCIS [6].

The risk should be minimized by a proper selection of patients, preoperative optimization, improving communication between anesthesiologist and surgeon, increasing the inspired oxygen concentration and avoiding intravascular volume depletion during cementing.

The management of BCIS is mostly supportive and includes use of $100 \%$ oxygen, fluids, vasopressors, pulmonary vasodilators, invasive monitoring, and intensive care $[1,3,4,6]$.

In high-risk patients, the use of non-cemented prosthesis, use of short-stem prosthesis, low-viscosity cement, retrograde application of the cement, and avoidance of excessive pressure may be helpful strategies to reduce the risk of developing BCIS [6].

Hip fracture surgeries using cemented prosthesis are associated with approximately $20 \%$ more adverse cardiovascular events compared with non-cemented prosthesis $[6,7]$. Nonetheless, the use of cemented prostheses for hip fracture surgery increases the likelihood of pain-free mobility after surgery [8], reduces the risk of re-operation and is associated with a lower mortality rate at 30 days [6].

There is no evidence that spinal anesthesia is better than general anesthesia and a retrospective analysis of a 65,535-patient national dataset did not find any significant difference in either a five-day or a 30-day postoperative mortality between general and spinal anesthesia [9]. In our case, we opted for single-shot spinal anesthesia, which is routinely performed as anesthetic technique for hip fracture in older patients, with a good outcome in the procedures conducted in our hospital.

The BCIS is a time-limited phenomenon, and high PVR usually normalizes within $24-48$ hours. Healthy patients may recover quickly from the insult, while others with risk factors, can present with a tragic manifestation resulting in death. Nevertheless, there exist many cases of patients without risk factors who died in consequence of BCIS. Early and aggressive resuscitation as well as surgical operative modifications may be the key to the prevention of this catastrophic syndrome. We suggest that a cemented prosthesis should be avoided in patients who are at high risk of BCIS unless there are overriding orthopedic considerations, as well as it should be considered the use of cemented prostheses in view of their risk and benefits. The final decision should be made by a multidisciplinary team.

\section{CONCLUSION}

Bone cement implantation syndrome is a significant cause of morbidity and mortality in orthopedic surgery. High index of suspicion and close monitoring is required at the time of cement insertion for early clinical diagnosis and outcome improvement. In selected patients, treatment with non-cemented prosthesis or a conservative approach of the fracture may reduce the mortality and so its potential risks and benefits compared with cemented arthroplasty should be pondered. This highlights the need for individualized medical team decision for each patient.

$* * * * * * * * *$

\section{Author Contributions}

Vera Barbosa - Substantial contributions to conception and design, Acquisition of data, Analysis and interpretation of data, Drafting the article, Revising it critically for important intellectual content, Final approval of the version to be published

Sandra Carneiro - Substantial contributions to conception and design, Acquisition of data, Analysis and interpretation of data, Drafting the article, Revising it critically for important intellectual content, Final approval of the version to be published

Joana Barros - Substantial contributions to conception and design, Acquisition of data, Analysis and interpretation of data, Drafting the article, Revising it critically for important intellectual content, Final approval of the version to be published

Teresa Rebelo - Substantial contributions to conception and design, Acquisition of data, Analysis and interpretation of data, Drafting the article, Revising 
it critically for important intellectual content, Final approval of the version to be published

\section{Guarantor}

The corresponding author is the guarantor of submission.

\section{Conflict of Interest}

Authors declare no conflict of interest.

\section{Copyright}

(C) 2016 Vera Barbosa et al. This article is distributed under the terms of Creative Commons Attribution License which permits unrestricted use, distribution and reproduction in any medium provided the original author(s) and original publisher are properly credited. Please see the copyright policy on the journal website for more information.

\section{REFERENCES}

1. Khanna G, Cernovsky J. Bone cement and the implication for anaesthesia. Contin Educ Anaesth Crit Care Pain 2012;12(4):213-6.

2. Donaldson AJ, Thomson HE, Harper NJ, Kenny NW. Bone cement implantation syndrome. Br J Anaesth 2009 Jan;102(1):12-22.

3. Rothberg DL, Kubiak EN, Peters CL, Randall RL, Aoki SK. Reducing the risk of bone cement implantation syndrome during femoral arthroplasty. Orthopedics 2013 Apr;36(4):e463-7.

4. Govil P, Kakar PN, Arora D, et al. Bone cement implantation syndrome: A report of four cases. Indian J Anaesth 2009 Apr;53(2):214-8.

5. Singh V, Bhakta P2, Zietak E3, Hussain A4. Bone cement implantation syndrome: a delayed postoperative presentation. J Clin Anesth 2016 Jun;31:274-7.

6. Griffiths R, White SM, Moppett IK, et al. Safety guideline: Reducing the risk from cemented hemiarthroplasty for hip fracture 2015: Association of Anaesthetists of Great Britain and Ireland British Orthopaedic Association British Geriatric Society. Anaesthesia 2015 May;70(5):623-6.

7. Olsen F, Kotyra M, Houltz E, Ricksten SE. Bone cement implantation syndrome in cemented hemiarthroplasty for femoral neck fracture: Incidence, risk factors, and effect on outcome. Br J Anaesth 2014 Nov;113(5):800-6.

8. Parker MJ, Gurusamy KS, Azegami S. Arthroplasties (with and without bone cement) for proximal femoral fractures in adults. Cochrane Database Syst Rev 2010 Jun 16;(6):CDoo1706.

9. White SM, Moppett IK, Griffiths R. Outcome by mode of anaesthesia for hip fracture surgery. An observational audit of 65535 patients in a national dataset. Anaesthesia 2014 Mar;69(3):224-30.
Access full text article on other devices

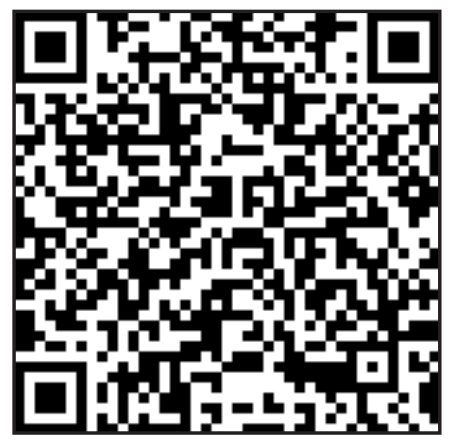

Access PDF of article on other devices

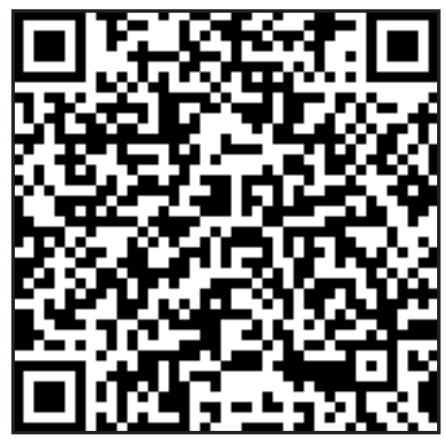

